\title{
A Semantic Learning Object (SLO)Web-Editor based on Web Ontology Language (OWL) using a New OWL2XSLO Approach
}

\author{
Zouhair Rimale \\ Dept. of Mathematics and computer \\ science \\ Faculty of Sciences Ben M'sik \\ Hassan II University \\ Casablanca, Morocco
}

\author{
EL Habib Benlahmar \\ Dept. of Mathematics and computer \\ science \\ Faculty of Sciences Ben M'sik \\ Hassan II University \\ Casablanca, Morocco
}

\author{
Abderrahim Tragha \\ Dept. of Mathematics and computer \\ science \\ Faculty of Sciences Ben M'sik \\ Hassan II University \\ Casablanca, Morocco
}

\begin{abstract}
Today, we see a strong demand for real-time information, with a rapid growth of m-learning. We also see that there are many educational resources on the Internet. Learning objects (LOs) are designed as a means of reusing these resources. Most of these LOs are built for e-learning systems based on desktop computers, which prevents their use on mobile devices. A LO is an area that is open to research and has a lot of potential in the creation, adaptation and production of learning content. There are standards that describe LOs in general as IEEE LOM, SCORM. Semantic web and its associated technologies are increasingly used in electronic document editing while separating the content from the presentation. Creating a LO with the semantic web is complex and raises difficulties because of the editing tools that require general knowledge of XML syntax and related technologies. In this paper, the authors propose a new OWL2XSLO approach based on ontologies (OWL) allowing the generation of XML-Schemas LOs. They then derive a semantic LO web editor based on OWL2XSLO approach for the generation of a content type enabling the editing of interactive LOs with XML technology and which can then be integrated into LMS and adapted to the mobile display.
\end{abstract}

Keywords-m-learning; learning objects; web semantic; XMLschema; xsd; owl; Ontology; rdf

\section{INTRODUCTION}

In recent years, m-learning has been boosted by the continued development of new information and communication technologies, which have become essential elements in education [1].

The design and development of teaching materials adapted to mobile devices may present difficulties for researchers and educators [2]. There is a wide variety of mobile devices and platforms; therefore, the pedagogical design of mobile content requires specific adjustments [3].

Educational content is numerous and consists of various types of media such as video, audio and presentations. Some of these LOs [4] are designed for fixed computers, which prevents their use on mobile devices. Among the problems that can occur are screen size and resolution, supported file type.

Teachers are looking for the best method to represent their courses. Since the LO becomes a smallest basic unit that can be designed to be reused, customized and adapted, teachers prefer to find creative tools to easily create interactive and adaptive LOs.

In order to implement an effective adaptive learning system, a new LO concept is needed. It is the separation of content from the presentation that could create and update a LO for different presentations [5] [6]. XML (Extended Markup Language) [7] is appropriate for implementing this separation. XML is the most promising technology to use specific parts of LOs to adapt them while using XSLT [8] style sheets.

In addition, the research studies related to the development of LOs, found that the ontology can be used to represent the knowledge in the development of LOs and able to support the semantic web development. Thus, the ontology plays an important role in creating standards for accessing to the contents and helpful for the learning management [9]. Moreover, the ontology is applied for constructing the personalized learning contents. The ontology approach can present the LOs, content structures and learning path that are corresponding to the learning environment of each individual [10]. It can also dynamically generate personalized hypertext relations powered by reasoning mechanisms over distributed RDF annotations [11].

In a digital learning environment a number of essential services are supported such as content creation that requires an educational content editor. The use of certain tools is not an easy task for anyone who wants to create specific educational content. In general, LMS / LCMS (Moodle [12], Dokeos [13], Atutor or Calorine [14]) have traditional editing tools. These tools are hypertext based with multimedia features for content creation in HTML format that is not suitable for direct display on small screens of mobile browsers. Importing content from external formats, even standardized (Microsoft Office, Open Office), pose several problems of compatibility, filtering and structuration.

At the present time, there are not really any editing tools that allow the creation of the basic LO adapted to the specific needs of the course designers by using the semantic web and from this the generation of the adaptive presentation to the display of mobile devices. The creation of the new LO with the 
semantic web raises difficulties because of the available tools that require a general knowledge of semantic web language and its related technologies such as XML technology.

In this paper, the authors propose a semantic LO web editor based on approach [15] called OWL2XSLO allowing the generation of the XML schema using two ontologies in order to create and share LOs with XML technology. The strength of the semantic LO web editor lies in its automatic generation of LO content types, which will allow organizations to organize, manage and process content in a coherent way. Beyond this synthesis, the semantic LO web editor wants to answer two fundamental questions about the creation of the new LO. The what? (What LO) and the how? (How the LO content will be represented).

\section{RELATED WORK}

Mobile devices offer new learning opportunities but their level of use in e-learning is still low [16]. E-learning systems and LMS have already been widely adopted to allow teachers to create learning materials [17]. For m-learning to be effective, it must be integrated with an existing e-learning platform in order to achieve better results regardless of the device used, including mobile devices [18] [16].

The important part of the interaction between the learner and a LO is the visual design of the LO. Mobile devices have several limitations, which also require specific adjustments. In earlier research, it was noted that learning content for mobile devices cannot be presented in the same way as e-learning used on computers because of the small size of the screen [16][19][20]. For the LMS to offer learning content suitable for all devices including mobile devices there should be a way for the teacher to define what to display on desktops and what to do Display on mobile devices.

In their research, Houser and Thornton [19] found that most educational web services can be modified to work on small screens. In the example of Houser and Thornton, a new course management system (CMS) called Poodle was developed as a step towards adopting the use of mobile phones in education. The Poodle system is a light version of the popular LMS Moodle, providing quizzes, a voting system, a wiki server and a flash card server to promote m-learning.

Bogdanović et al. [16] extended the Moodle platform with a mobile quiz application to study how students react to mlearning in a real context. The presented application works as a plug-and-play component that recognizes the device used and adapts the content using different CSS themes.

To support new pedagogies and technologies related to technology-enhanced learning, LOs have been developed as a new conceptual model for the creation and distribution of content [21]. Hodgins and Duval [22] defined LO as any numerical or non-numerical entity that can be used for learning, education and training. LOs are building blocks that can be combined in a virtually infinite way to build collections that can be called lessons, modules, and courses [23].

The most widely used standards for packaging and composition of LOs are IMS-LD and Moodle. It also allows for the exchange and re-use of learning design across platforms and supports a wide range of modern educational approaches such as active learning, collaborative learning, adaptive learning And skills-based learning [24].

The problem is that the creation tools based on IMS-LD require non-mobile platforms. As well as the IMS-LD does not have adequate mobility support and there is no widely accepted theory of m-learning. Some limitations are related to the physical aspects of mobile technologies.

Sotsenko et al. [25] discuss the use of LO in m-learning environments by adding contextual information as metadata to provide the learner with the right content at the right time to solve problems related to mobile devices such as small size screen. The idea is to obtain contextual information from the learner, using the detection mechanisms in the mobile device and the contextual information provided by the learning platform, and to provide the most appropriate content that corresponds to the personal context of the learner, the environmental context, as well as the properties of the device and the connection to the network.

Metadata is essential for processing implementations of the LO. This concept allows the discovery, retrieval and reuse of LOs [26]. In recent years, the number of LO repository has been developed, such as Merlot [27], Ariadne [28], Careo [29]. Most of these repositories focus on the e-learning perspective and there is no interoperability fully achieved with mobile devices.

According to Hodgins [21], the way LOs are to be used and sequenced is also very important. It also recalls that in order for LOs to be widely adopted, there must be tools that make the process of conceptualization, design, construction and selection quick and easy. It would be important to consider how the authors could create adaptive LOs that can be both used by existing LMS and integrated with LORs to support m-learning.

\section{THE PROPOSED OWL2XSLO APPROACH}

\section{A. Objective}

The purpose of the OWL2XSLO approach is to generate automatically from two ontologies, an XML-schema (XSD) to create LOs with XML technology using the web ontology language (OWL). Ontology is used to provide domain knowledge such as terminology, restrictions and data types. The OWL2XSLO approach can be used in all areas including notably education m-learning, provided that ontology is available for this area. This will have a big impact on applications such as communication systems where XML is widely used. Manual labor will be reduced in professional organizations including universities and schools on the creation of LOs in XML. Once the ontology is created for a university to the description of domain knowledge of the course for example, it is possible to refer the terms used in this ontology in the XML instance documents to automatically generate the XML-schema.

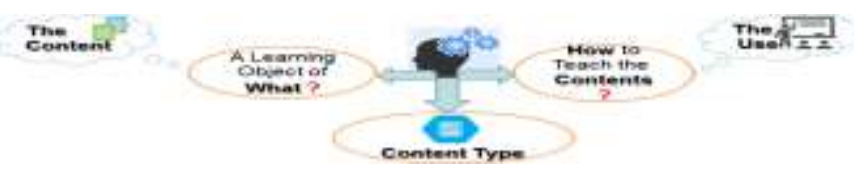

Fig. 1. The conceptual model approach «OWL2XSLO» 


\section{B. Methodology}

Most existing tools that allow the generation of the specific XML schema based on data in the XML document. Though the XML schema that can be generated, it will not be the most accurate diagram. The reason for this is mainly due to the lack of knowledge of the area in which the XML documents are created.

The authors use OWL2XSLO approach to overcome the above problems while providing successively two ontologies as mounted in Figure 2 below, ontology "Content Ontology" which represents the knowledge, skills, items and vocabularies an essential way to establish and develop a structure of the LO and the other ontology "Ontology of Use" which represents the know-how and allows to define how to use knowledge, skills that it necessary to include any LO (make definitions, make examples, do exercises and corrected).

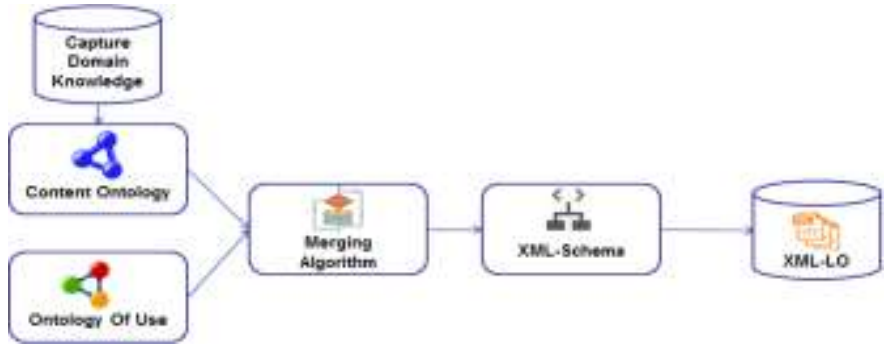

Fig. 2. The conceptual model OWL2XSLO approach

- The Content Ontology (What): This ontology is a structure of concepts that describe the knowledge domain. It consists of knowledge in the forms of the hierarchical data structure to describe the interested knowledge domain (Figure 3). The use of this ontology is adapted to the sharing of knowledge in the same knowledge. Thus, using this ontology for the design of LOs would be useful for providing knowledge and building a standardization of knowledge content that is also passed on to learners.

To automatically create an XML schema for each LO in $\mathrm{XML}$, it is necessary to store this ontology in a form readable by the machine by using the OWL. The Ontology Web Language (OWL) is based on the RDF (Resource Description Framework) [30]. Then the SPARQL (SPARQL protocol and RDF Query Language) is applied to query the information in a RDF format [31].

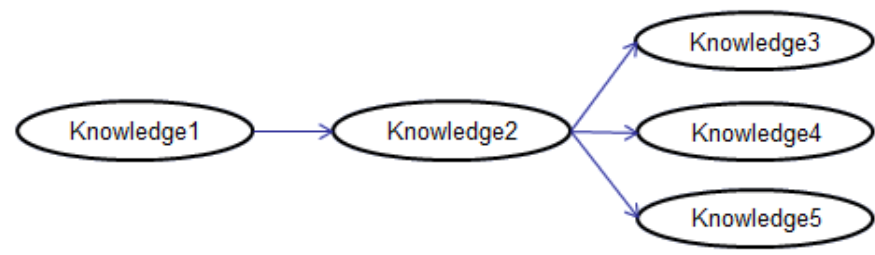

Fig. 3. Content Ontology

- Ontology of Use (How): The lesson plan is for the teacher a communication tool of his intentions, his expectations and the requirements of the course. Its quality rests on the consistency with the course itself, the accuracy and relevance of its content. The syllabus allows the professor to have a faithful portrait of his course, to confirm the structure of the course and to communicate the essential elements to the persons concerned, namely the students. For the professor, writing and revising his lesson plans are opportunities to check the division into modules or lessons, to re-evaluate the time allocated to the most important objectives, to adapt or modify his course. But the course plan is also a course management tool. It makes it possible to better control its progress and to avoid deviations or delays that are detrimental to students.

As shown in Figure 4, ontology of use aims to describe how each concept (knowledge) of the content ontology will be presented in order to create a coherent LO plan.

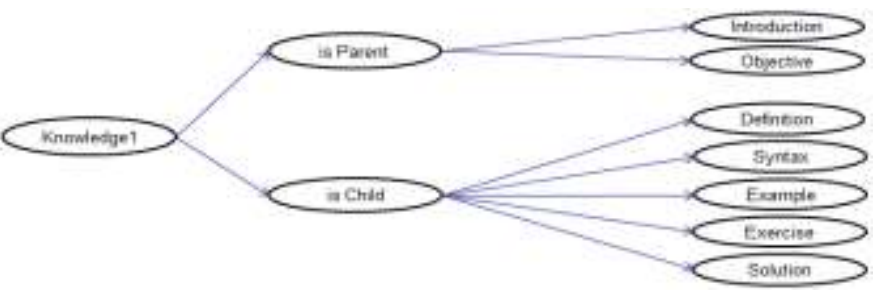

Fig. 4. Ontology of Use

- Ontology Merging Algorithm: After setting the Framework of ontology, the next step was the development of the algorithm as shown in Figure 6. The objective of the algorithm was to automatically merging two ontologies to create a single coherent ontology, or (2) aligning the ontologies by establishing links between them and allowing them to reuse information from one another. The process of the algorithm takes as input two (or more) source ontologies and returns a merged ontology based on the given source ontologies as shown in figure 5 .

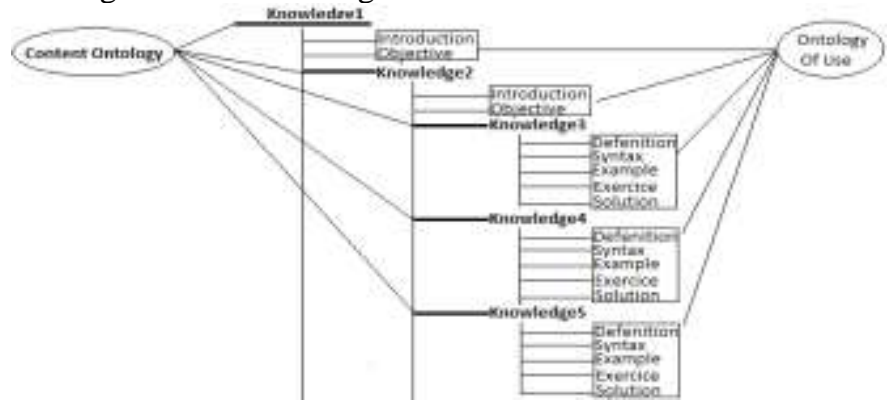

Fig. 5. Process of Ontology Algorithm Merging [15]

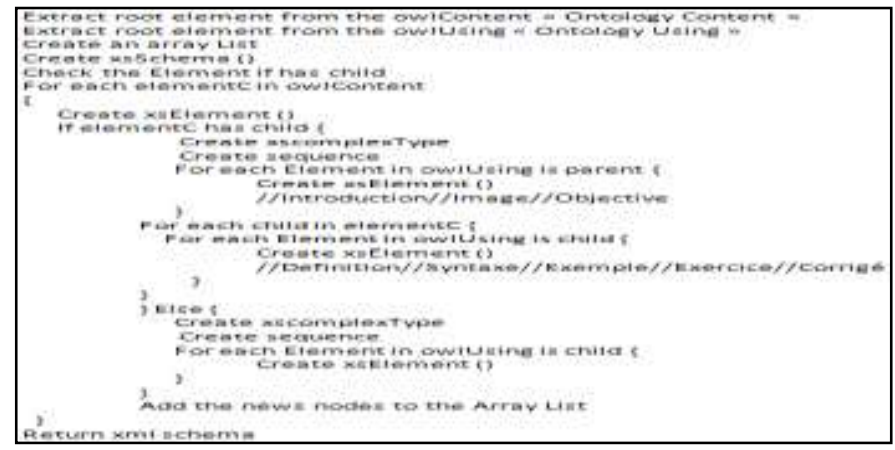

Fig. 6. Algorithm of the OWL2XSLO approch [15] 
The algorithm of the OWL2XSLO approach allows the automatic generation of the XML schema (Figure 9) from two ontologies fused above (using the OWL) while successively using two conversions, basic and advanced as shown the two figures below 7 and 8 . The algorithm takes each knowledge of 'Content Ontology' by checking whether it is parent or child element. If the selected knowledge is a parent element then a complex element should be created with the elements of the parent type of "ontology of use". If not a simple element should be created with the elements of child type of "ontology of use".

\begin{tabular}{|c|c|}
\hline $\begin{array}{l}\text { ow1:C1ans } \\
\text { ow } 1 \text { :Datatypeproperty }\end{array}$ & xad:aimpleType \\
\hline $\begin{array}{l}\text { ow1: Clans } \\
\text { ow1: Objectproperty }\end{array}$ & $\begin{array}{l}\text { Kad: aimple TYpe } \\
\text { nad: compl lexтуре }\end{array}$ \\
\hline owl: Restriction & $\begin{array}{l}\text { xad: restriction } \\
\text { xad: enumeration } \\
\text { min-/maxoecura }\end{array}$ \\
\hline
\end{tabular}

Fig. 7. Basic Conversion [15]

\begin{tabular}{|c|c|}
\hline rdfs: subclassof $\longrightarrow$ & $\begin{array}{l}\text { xsd: complexContent } \\
\text { xsd: restriction base } \\
\text { substitutionGroup } \\
\text { abstract }\end{array}$ \\
\hline
\end{tabular}

Fig. 8. Advanced Conversion [15]

\section{Proposal: New SEMAntic Lo Web Editor}

\section{A. OverView of the semantic $L O$ web editor}

The semantic LO web editor is a creative environment based on the semantic web using the ontology web language (OWL) and XML technology. It is designed to help teachers and academics in the design, development and publication of interactive LOs without the need to become proficient in XML.

The Semantic Web is a revolutionary educational tool because it presents to teachers and learners a technology that simultaneously provides content with the means of adaptation. Unfortunately, the power of the semantic web is restricted in educational settings because the vast majority of teachers and academics do not have the technical skills to build their own LOs. The semantic LO web editor is under development to overcome a number of identified limitations:

- Traditionally, existing creative tools require general semantic web knowledge, they have not been intuitive and they were not designed for publishing learning content. As a result, teachers and academics have not adopted these technologies for the publication of LO content. The semantic LO web editor aims to provide an intuitive and easy-to-use tool that will allow teachers to publish interactive LOs and can adapt for m-learning.

- Currently, LMSs do not offer sophisticated creation tools to create LO content with XML technology. The publisher is a tool that offers professional LO publishing features that will be easily referenced or imported by LMS standards;

- Many LMS do not provide an intuitive WYSIWYG environment allowing the creation of LOs with XML technology. The semantic LO web editor mimics the WYSIWYG feature that allows authors to see what content will look like when they are published online.

With the semantic LO web editor, authors will have a content type suited to their needs enabling the construction of the flexible and easily updated LO.

\section{B. Architecture of the Semantic Learning Object Web-Editor}

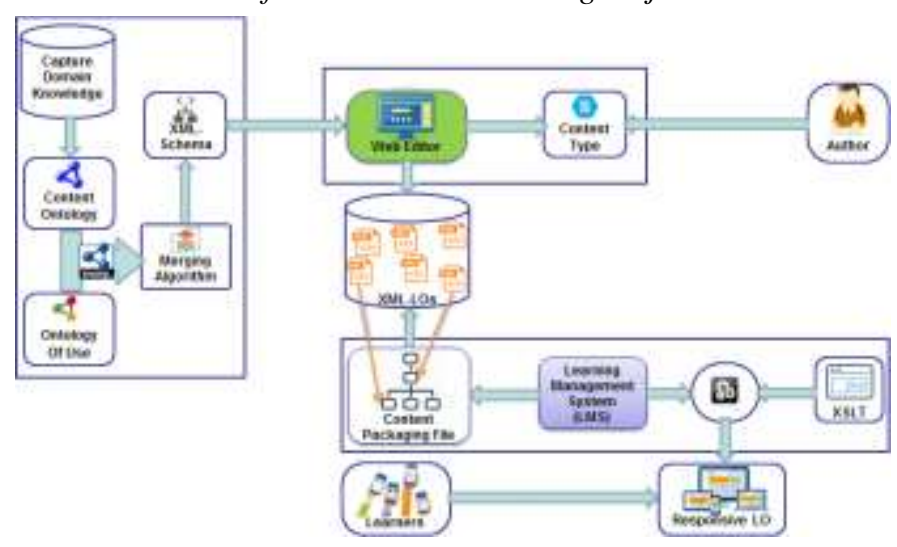

Fig. 9. Architecture of the semantic LO web editor

The architecture of the semantic LO web editor is based on 4 essential elements:

An environment based on the OWL2XSLO approach allowing the automatic generation of the XML-schema using OWL and SPARQL.

A semantic XML environment based on a content type allowing the creation, storage, modification and dissemination of LOs in XML.

A storage environment for LOs in a single repository, to separate the contents from its presentation.

An LCMS / LMS environment enabling authors to create, store, reuse, manage and distribute LOs to associate them, order them to build a coherent course. This solution is based on the choice of free software that complies with the postulates of the LCMS.

An environment for adapting semantic XML content in a format suitable for displaying mobile devices using XSLT.

The design can be presented in the following scenario:

The course author can modify and create his LOs using a web content type (web form) that is generated automatically by respecting an XML-schema. The XML-Schema (figure 11) based on OWL2XSLO approach is also automatically generated by successively using two ontologies, the "SELECT" knowledge of the "content ontology" that allows the representation of the SQL language knowledge [9] as shown on figure 10 and "ontology of use" as shown on figure 4. The storage of these LOs is possible in their original format (XML) conforming to the metadata structure defined in an XML schema format [32] (FIG. 9). Using an LMS, the author can associate and order these LOs in order to build a coherent course. Using the XSLT transformations and the CSS3, an LO with its XML content can have several presentations generated on demand in formats suitable for displaying mobile devices. 


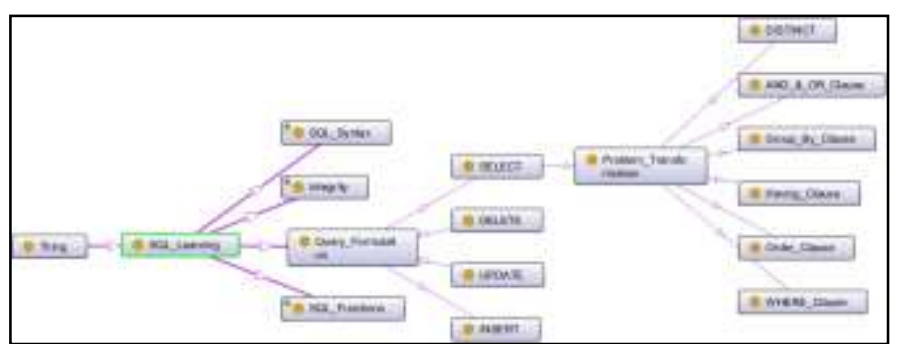

Fig. 10. Content Ontology: SQL Knowledge Domaine[9]

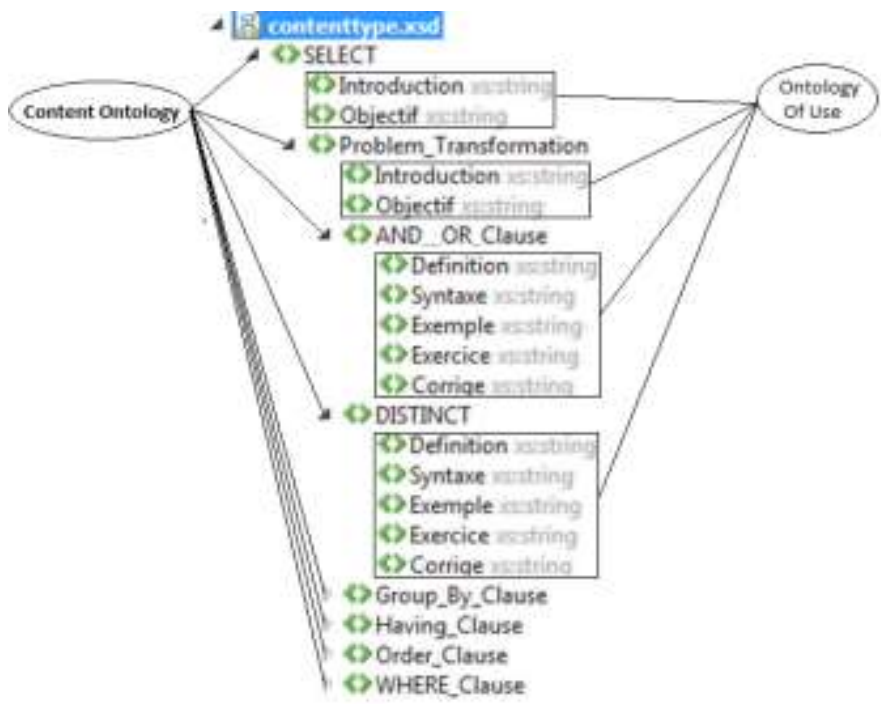

Fig. 11. Result of the OWL2XSLO approach : XML-Schema of Knowledge "SELECT"

\section{Implementation of the Semantic LO Web Editor}

For the validation of the proposal, the web editor is developed around ontologies and the Microsoft .Net on a Windows 7 platform. We used Protege [33] for the construction of ontologies with the ontology web language (OWL), The SPARQL query language to query and query the OWL, Microsoft Visual Studio 2015 [34] to create the platform using Microsoft ASP.NET (Active Server Page) web technology. The learning module chosen for validation is the SQL [9] language. To use of the semantic LO web editor we need an Internet Browser only without installation or update.

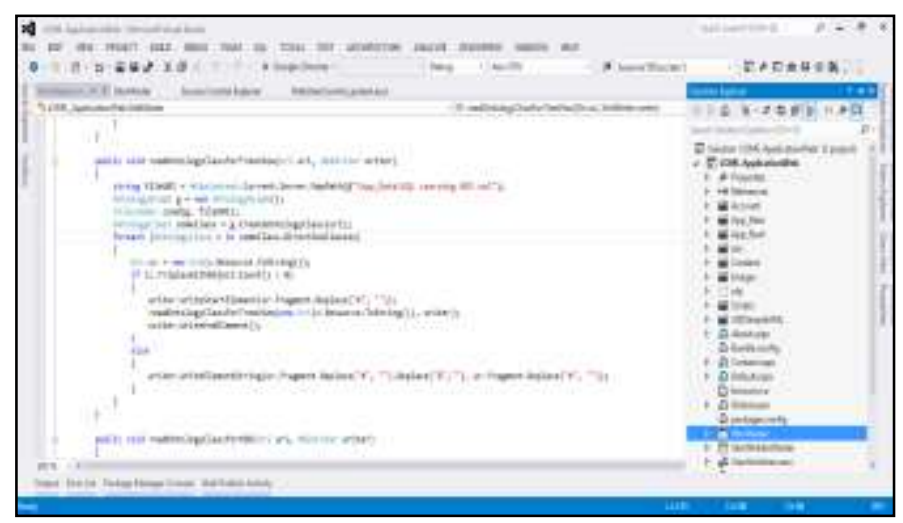

Fig. 12. Technical Architecture under micrsoft visual strudio 2015[34]
The user interface comprises different parts, each having a special role:

1) Search box: Allows the author to search, modify or create a new content type of LO.

2) The Tree View to the left: represents the plan, the main elements and the tree of the LO

3) The editing area: area where the author can create the content of the LO using the text areas.

4) Navigation buttons: allows the author to save and display the next or previous element of the LO.

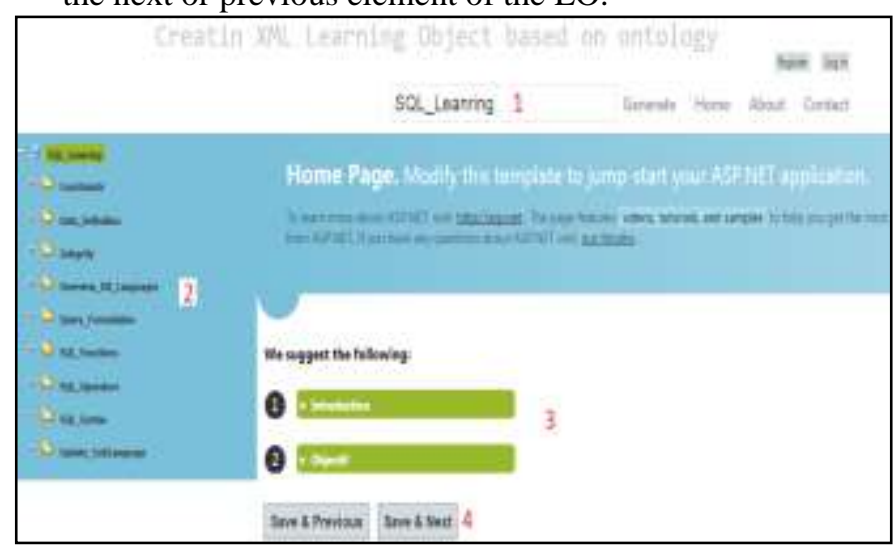

Fig. 13. User Interface of the Semantic LO Web Editor

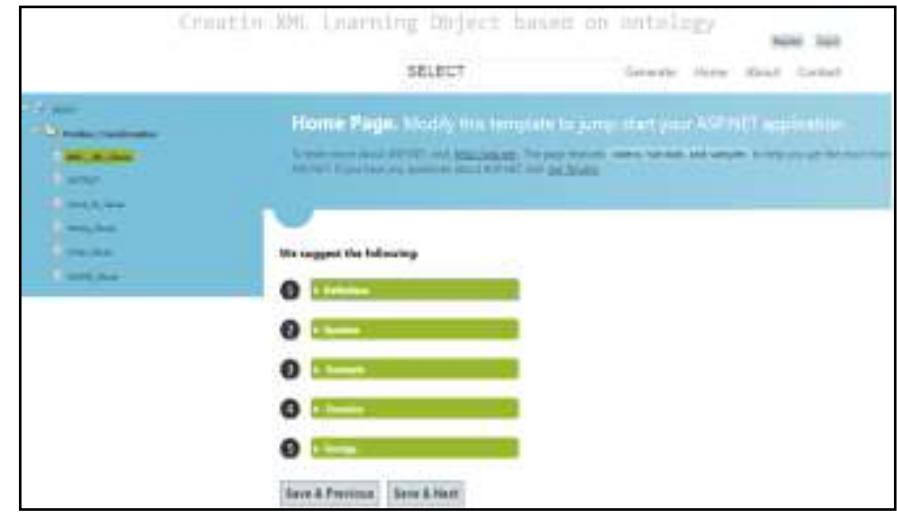

Fig. 14. User interface of the LO content type "SELECT"

\section{CONCLUSION AND FUTURE WORK}

This article presents on the one hand the conception of the original approach titled OWL2XSLO allowing the generation of the XML-schema of learning object from two ontologies and on the other hand the construction of the semantic LO web editor allows the generation of the LO content type (web form) from this XML-schema.

For the future work, the authors describe another approach based on the web services for the integration of an environment of adaptation of a pedagogical content for a mobile use. As a result, they can set up a multimodal content adaptation system for a mobile learning environment. They can adopt web services technology to provide a flexible integration model in which all components of learning and applications will be well defined. The future work has the advantage of using a mobile web browser as a universal communication environment. 


\section{REFERENCES}

[1] Zouhair Rimale, El Habib Ben Lahmar, Abderrahim Tragha, A Brief Survey and Comparison of m-Learning and e-Learning, International Journal of Computer Networks and Communications. Vol.4, Issue 4, April 2016

[2] Alvarez, C., Alarcon, R., Nussbaum, M.. Implementing collaborative learning activities in the classroom supported by one-to-one mobile computing: A design-based process, Journal of Systems and Softwar, Vol. 84, 2011. p. 1961-1976. doi:10.1016/j.jss.2011.07.011.

[3] Güler, Ç., Kılıç, E., Çavuş, H. A comparison of difficulties in instructional design processes: Mobile vs. desktop, Computer Human Behavior, Vol. 39, 2014. p. 128-135. doi:10.1016/j.chb.2014.07.008.

[4] Moreno JER (2012) Adaptation of learning strategies in learning objects for using learning styles. IERI Procedia 2:808814.doi:10.1016/j.ieri.2012.06.175

[5] Parupalli R, Nelaturu SCB, Jain DK (2011) The role of content adaptation in ubiquitous learning. In: 2011 IEEE international conference on technology for education, pp 177-182. doi:10. 1109/T4E.2011.35

[6] Mohan, P., \& Greer, J. (2003, June). Reusable learning objects: Current status and future directions. Paper presented at ED-MEDIA 2003 World Conference on Educational Multimedia. Hypermedia \& Telecommunications, Honolulu, Hawaii. Retrieved January 3, 2006, from

http://www.campussaskatchewan.ca/pdf.asp?pdf=PMohanEdMedia03.p df

[7] Extensible Markup Language (XML). http://www.w3.org/XML.

[8] Extended Style Sheet Transformations http://www.w3.org/TR/xslt

[9] Wilairat Yathongchai, Thara Angskun, and Jitimon Angskun "SQL Learning Object Ontology for an Intelligent Tutoring System "Internation Journal of e-Education, e-Business, e-Management and eLearning, Vol. 3, No. 2, April 2013

[10] J. Jovanovic, D. Gašević, and V. Devedžić, "TANGRAM for Personalized Learning Using the Semantic Web Technologies," Journal of Emerging Technologies in Web Intelligence, vol. 1, no 1, 2009, pp. 621.

[11] N. Henze, P. Dolog, and W. Nejdl, "Reasoning and Ontologies for Personalized E-Learning in the Semantic Web," Educational Technology \& Society, vol. 7 no 4, 2004, pp. 82-97.

[12] Moodle open-source community-based tools for leanring http://moodle.org

[13] Dokeos: e-learing open source, http://www.dokeos.com

[14] Atutor learning Management System, http://www.atutor.ca

[15] Zouhair Rimale, El Habib Ben Lahmar, Abderrahim Tragha, (2016) An Approach for the Automatic Generation af a Content Type of a Semantic Learning Object from Ontology. In: 2016 ieee $11^{\text {th }}$ International Conference On Intelligent Systems : theories and Applications.

[16] [Bogdanović et al., 2014] Bogdanović, Z., Barać, D., Jovanić, B., Popović, S., and Radenković, B., Evaluation of mobile assessment in a learning management system. British Journal of Educational Technology, 45 (2014), 231-244.

[17] [Ako-Nai and Tan, 2013] Ako-Nai, F. and Tan, Q. Location-Based Learning Management System for Adaptive Mobile Learning.
International Journal of Information and Education Technology 3, 5 (2013), 529-535.

[18] [Fayed et al., 2013] Fayed, I., Yacoub, A., and Hussein, A. Exploring the impact of using tablet devices in enhancing students listening and speaking skills in tertiary 93 education. QScience Proceedings: Vol. 2013, 12th World Conference on Mobile and Contextual Learning (mLearn 2013), 1.

[19] [Houser and Thornton, 2005] Houser, C. and Thornton, P., "Poodle: a coursemanagement system for mobile phones," Wireless and Mobile Technologies in Education, 2005. WMTE 2005. IEEE International Workshop on Wireless and Mobile Technologies in Education (Nov. 2005), 28-30.

[20] [Shih et al., 2011] Shih, J.-L., Chu, H.-C., Hwang, G.-J., and Kinshuk. An investigation of attitudes of students and teachers about participating in a context-aware ubiquitous learning activity. British Journal of Educational Technology, 42, 3 (2011), 373-394.

[21] [Hodgins, 2002] Hodgins, H.W. The Future of Learning Objects. In: 2002 ECI Conference on e-Technologies in Engineering Education: Learning Outcomes Providing Future Possibilities. Lohmann, J.R., Georgia Institute of Technology, USA, Corradini, M.L., University of Wisconsin-Madison, USA (Eds.). ECI Symposium Series, P01 (2002).

[22] Hodgins W., Duval E. 1484.12.1-2002 - IEEE Standard for Learning Object $\quad 2002 . \quad$ Retrieved 20.10.2014, URL:http://ieeexplore.ieee.org/stamp/stamp.jsp?tp=\&arnumber=103284 3\&isnumber $=22180$.

[23] Chikh, A. A general model of learning design objects, Journal of King Saud Univeristy - Computer and Information Sciences, Vol. 26, 2014. p. 29-40. doi:10.1016/j.jksuci.2013.03.001.

[24] Koper, R., Olivier, B. Representing the Learning Design of Units of Learning, Educational Technology\&Society, Vol. 7; 2004. p. 97-111

[25] [Sotsenko et al., 2013] Sotsenko, J., Jansen, M., and Milrad, M. About the Contextualization of Learning Objects in Mobile Learning Settings. QScience Proceedings: Vol. 2013, 12th World Conference on Mobile and Contextual Learning (mLearn 2013), article 11 (Oct. 2013), 67-70.

[26] Roy, D., Sarkar, S., Ghose, S. A Comparative Study of Learning Object Metadata, Learning Material Repositories , Metadata Annotation \& an Automatic Metadata Annotation Tool, Vol. 2, 2010. p. 103-126.

[27] Merlot, Multimedia Educational Resource for Learning and Online Teaching, 2009. Retrieved 12.10.2014, URL: http://www.merlot.org.

[28] Aradne Foundation, ARIADNE, 2014. Retrieved 12.10.2014, URL: http://ariadne-eu.org/.

[29] Careo project, Campus Alberta Repository of Educational Objects, 2014. . Retrieved 12.10.2014, URL: http://careo.org/

[30] W3C. (2004). RDF Vocabulary Description Language 1.0: RDF Schema. W3C Recommendation. [Online]. Available: http://www.w3.org/TR/rdf-schema/.

[31] W3C. SPARQL Query Language for RDF. [Online]. Available:http://www.w3.org/TR/rdf-sparql-query

[32] XML Schema, http://www.w3.org/xml/schema

[33] webprotege [http://webprotege.stanford.edu/

[34] Microsoft Visual Studio 2015. https://www.visualstudio.com/ 